04

\title{
Корреляция выхода нейтронов из плазменного фокуса с изменением энергии магнитного поля разрядного контура
}

\author{
(C) В.Е. Аблесимов, Ю.Н. Долин, А.Е. Калинычев, З.С. Цибиков
}

Российский фредеральный ядерный центр - Всероссийский научно-исследовательский институт экспериментальной физики,

607188 Саров, Нижегородская обл., Россия

e-mail: ablesimov.v@Gmail.com

(Поступило в Редакцию 8 ноября 2016 г.)

Для разрядной плазмофокусной камеры мейзеровского типа исследована связь выхода нейтронов $Y$ с величиной изменения энергии $\Delta W$ магнитного поля разрядного контура. Интегральный выход ДД-нейтронов источника измерялся активационной методикой (активация изотопов серебра). Временна́я зависимость выхода нейтронов регистрировалась сцинтилляционными детекторами. Для исследованной установки обнаружена линейная связь выхода нейтронов с величиной изменения энергии $\Delta W$ магнитного поля разрядного контура в момент генерации нейтронов, а также влияние на указанную связь величины начального давления дейтерия в разрядной камере.

DOI: $10.21883 / J T F .2017 .10 .44993 .2090$

\section{Введение}

Для установок плазмофокусного (ПФ) типа существует известная зависимость выхода нейтронов $Y$ от тока разряда в момент пинчевания $I_{p}: Y \sim I_{p}^{4}$. Это соотношение позволяет сравнивать и прогнозировать характеристики различных вариантов устройств подобного типа.

Практически во всех исследованиях процессов, происходящих в разряде типа ПФ, отмечается, что выход нейтронов увеличивается с величиной скачка разрядного тока в момент пинчевания (так называемой особенности). Исследованию корреляции происходящих в плазмофокусной камере процессов с величиной выхода нейтронов посвящен ряд работ, выполненных в последние годы с целью более глубокого понимания механизма генерации нейтронов и поиска возможных путей увеличения их выхода $[1,2]$. В частности, в работе [1] показано, что существует сильная корреляция между интенсивностью пучка ионов, зарегистрированной камерой Фарадея, и скачком тока в радиальной фазе разряда.

Мы провели измерения выхода нейтронов для двух отличающихся конструкций камер при практически неизменной (с точностью $1-2 \%$ ) величине тока разряда. Результаты этих измерений, изложенные в работах $[3,4]$, обнаруживают корреляцию величины выхода нейтронов с величиной скачка разрядного тока, который в свою очередь связан с изменением магнитного поля разрядного контура.

В конечном счете, характеристики ПФ устройств (в том числе излучательные) должны определяться диссипацией магнитной энергии в процессе разряда.

Энергия магнитного поля разрядного контура связана с величиной тока соотношением

$$
W(t)=1 / 2 L I^{2} .
$$

Напряжение между электродами камеры $U=$ $=d(L I) / d t+R I$, причем, как правило, резистивным членом можно пренебречь по сравнению с индуктивным слагаемым. Отсюда $d(L I)=U(t) d t$,

$$
L(t) I(t)=\int_{0}^{t} U(t) d t .
$$

Выражая отсюда $L(t)$ и подставляя в $(1)$, получим

$$
W(t)=1 / 2 I(t) \int_{0}^{t} U(t) d t .
$$

В выражение (2) входят зависимости электрических характеристик разряда от времени, измеряемые в процессе эксперимента.

\section{Постановка измерений}

В настоящей работе исследована связь выхода нейтронов $Y$ с величиной изменения энергии $\Delta W$ магнитного поля разрядного контура. Интегральный выход ДД-нейтронов источника измерялся активационной методикой (активация изотопов серебра). Временна́я зависимость выхода нейтронов регистрировалась сцинтилляционными детекторами.

Камера размещалась на установке МОДУЛь [5]. Установка представляет собой импульсную конденсаторную батарею и состоит из двух одинаковых энергетических секций емкостью $18 \mu \mathrm{F}$ каждая. Емкость конденсаторной батареи установки МОДУЛЬ равняется $36 \mu \mathrm{F}$, напряжение, подаваемое на камеру, составляло $27 \mathrm{kV}$, энергозапас установки около $13 \mathrm{~kJ}$.

Использовалась плазмофокусная разрядная камера с длинными $(l>d)$ цилиндрическими коаксиальными 


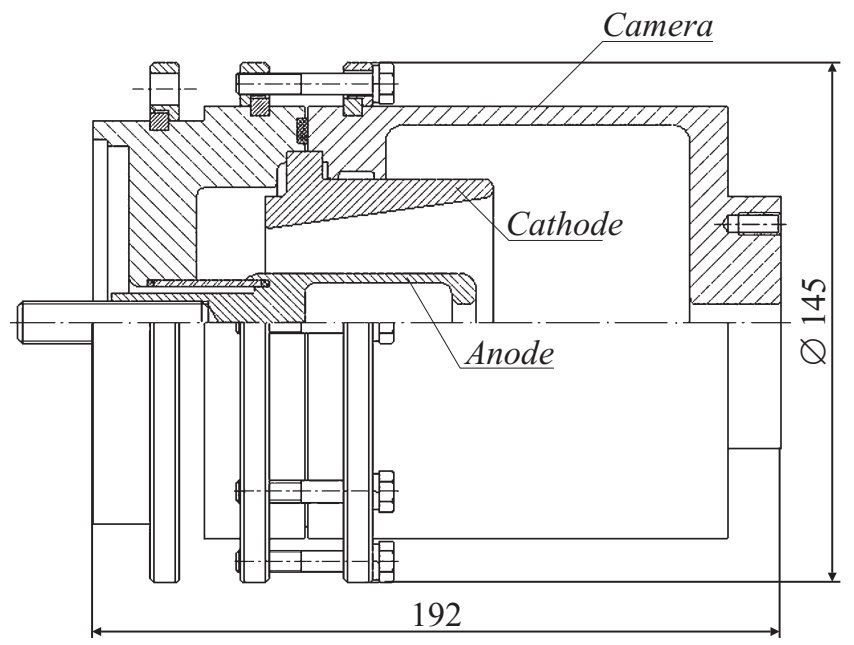

Рис. 1. Электродная система плазмофокусной камеры.

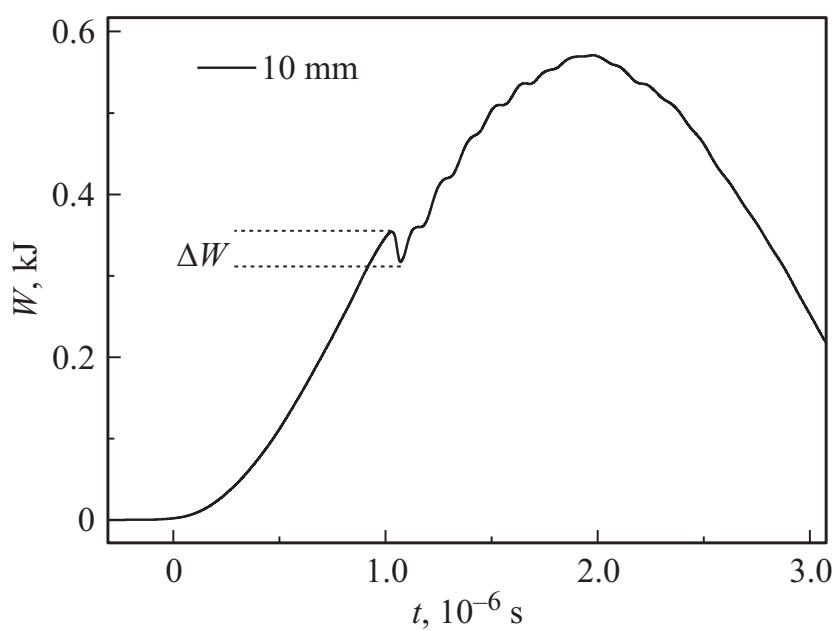

Рис. 2. Зависимость энергии магнитного поля разрядного контура от времени $P=10^{3} \mathrm{~Pa}$.

электродами, так называемая „мейзеровская“ камера [6]. Потенциальный электрод — анод, диаметром $27.5 \mathrm{~mm}$. Заземленный электрод - катод состоит из двух секций: нижняя часть выполнена в виде трубы $\varnothing 75 \mathrm{~mm}$ и высотой $19 \mathrm{~mm}$; верхняя секция в виде расходящегося конуса с диаметром от 52 до $70 \mathrm{~mm}$ и высотой $63.5 \mathrm{~mm}$. Торцевое окончания анода находится на расстоянии $77.5 \mathrm{~mm}$ от входного заземленного фланца. Анод и входной фланец разделены между собой керамическим изолятором. Диаметр изолятора $24 \mathrm{~mm}$, высота $16 \mathrm{~mm}$. Электродная система камеры располагается в герметичном корпусе. Конструкция камеры приведена на рис. 1. Используются неорганические вакуумные уплотнения, откачка осуществляется безмасляным турбомолекулярным насосом. С этой же камерой были проведены исследования, описанные в [3].

Напряжение на нагрузке. При помощи „пояса Роговского“ измерялся ток, проходящий через сопротивление
$R_{s}=165 \Omega$, установленное параллельно нагрузке $(П \Phi)$ и индуктивности токосборника. Электрические характеристики системы измерения тока: $R_{H}=2.27 \Omega-$ сопротивление нагрузки пояса; $L_{H} \approx 2 \mathrm{nH}-$ индуктивность нагрузки пояса ; $R_{\mathrm{B}}=0.13 \Omega-$ активное сопротивление катушки; $L \mathrm{~B}=402 \mu \mathrm{H}$ - индуктивность катушки. По данным с токового датчика вычисляется напряжение на нагрузке.

Измерение производной тока разряда. Измерительный „пояс“ представляет собой катушку с $N=3$ витками из проводника диаметром $0.22 \mathrm{~mm}$, намотанного на каркас из текстолита в виде тора со средним диаметром $\varnothing 124.4 \mathrm{~mm}$ и квадратным сечением $3.4 \times 3.4 \mathrm{~mm}$. Электрические характеристики системы измерения тока: $R_{H}=75 \Omega-$ сопротивление нагрузки пояса; $L_{H} \approx 2 \mathrm{nH}-$ индуктивность нагрузки пояса; $R_{\mathrm{B}}=0.2 \Omega$ - активное сопротивление катушки; $L_{\mathrm{B}}=10 \mathrm{nH}-$ индуктивность катушки. Ток разряда определялся интегрированием сигнала производной.

\section{Результаты измерений}

Нами проведено 4 серии измерений по 15-20 импульсов для 4 значений давления дейтерия, наполняющего камеру, $-(5,10,18,27) \cdot 10^{2} \mathrm{~Pa}$. Внутри каждой серии ток разряда остается практически неизменным.

Суммарные результаты проведенных измерений приведены в таблице.

Сводка результатов измерений

\begin{tabular}{c|c|c|c|c|c}
\hline $\begin{array}{c}P, \\
\times 10^{2} \mathrm{~Pa}\end{array}$ & $\begin{array}{c}Y, \\
\times 10^{9} \mathrm{n} / \mathrm{imp}\end{array}$ & $\begin{array}{c}\Delta Y, \\
\times 10^{9} \mathrm{n} / \mathrm{imp}\end{array}$ & $\begin{array}{c}I, \\
\mathrm{kA}\end{array}$ & $\begin{array}{c}\Delta I, \\
\%\end{array}$ & $\begin{array}{c}\Delta W, \\
\mathrm{~kJ}\end{array}$ \\
\hline 5 & 0.67 & 0.16 & 242 & 1.7 & 0.016 \\
10 & 1.98 & 0.5 & 295 & 2 & 0.043 \\
18 & 2.32 & 0.84 & 316 & 3 & 0.040 \\
27 & 2.48 & 0.67 & 335 & 1 & 0.054
\end{tabular}

Примечание. Здесь $P$ - давление дейтерия в камере, $Y, \Delta Y-$ средний по серии выход нейтронов в импульсе $(\mathrm{n} / \mathrm{imp})$ и его погрешность, $I, \Delta I$ - максимальный ток в импульсе и его погрешность, $\Delta W-$ изменение энергии магнитного поля разрядного контура.

По результатам измерений были построены зависимости $W(t)$, пример обработки которых приведен на рис 2, определено значение изменения $\Delta W(t)$ для каждого импульса, которое затем поставлено в соответствии с величиной интегрального выхода нейтронов в соответствующем импульсе.

Зависимость величины интегрального выхода нейтронов $Y$ в соответствующем импульсе от значения изменения энергии $\Delta W$ магнитного поля разрядного контура обнаруживает значимую корреляцию (рис. 3-6). С ростом давления эта корреляция несколько ослабляется. 


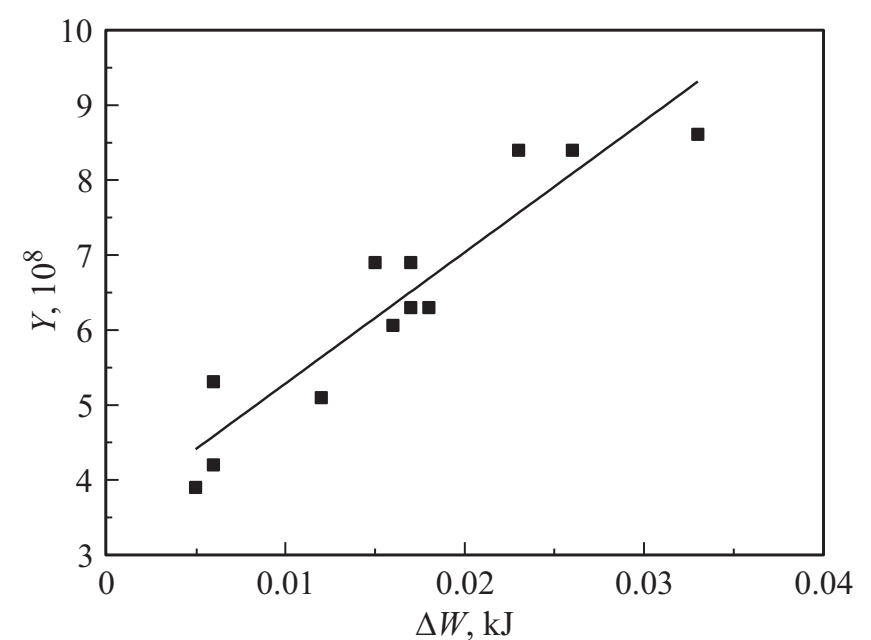

Рис. 3. Зависимость выхода нейтронов $Y$ от изменения энергии $\Delta W$ магнитного поля, $P=5 \cdot 10^{2} \mathrm{~Pa}$, коэффициент корреляции 0.93 .

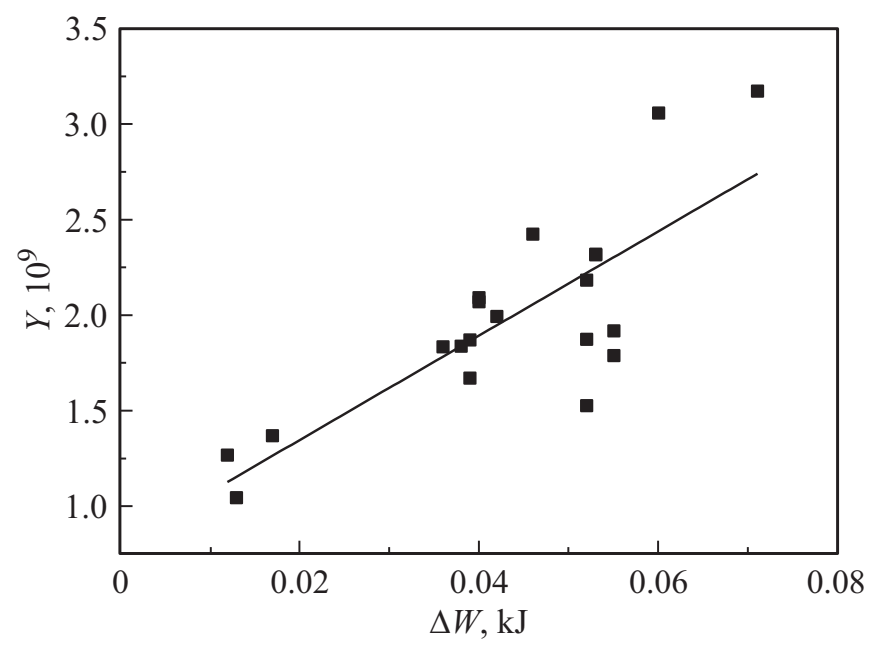

Рис. 4. Зависимость выхода нейтронов $Y$ от изменения энергии $\Delta W$ магнитного поля, $P=10^{3} \mathrm{~Pa}$, коэффициент корреляции 0.79 .

\section{Выводы}

Результаты проведенной таким образом обработки экспериментальных данных обнаруживают значимую корреляцию выхода $Y$ с величиной изменения магнитной энергии разрядного тока. Кроме того, обнаружено, что величина коэффициента корреляции зависит от начального давления наполняющего камеру дейтерия.

Следует отметить, что величина $\Delta W$ составляет малую часть $\sim 0.5 \%$ от запасенной энергии КБ. Нейтронное излучение в приближении изотропного выхода $Y=2.5 \cdot 10^{9} \mathrm{n} /$ puls уносит энергию $\sim 10^{-3} \mathrm{~J}$. Остальная часть переходит в энергию пучков заряженных частиц, ударной волны и в конечном счете в рассеивающуюся в пространстве тепловую энергию.

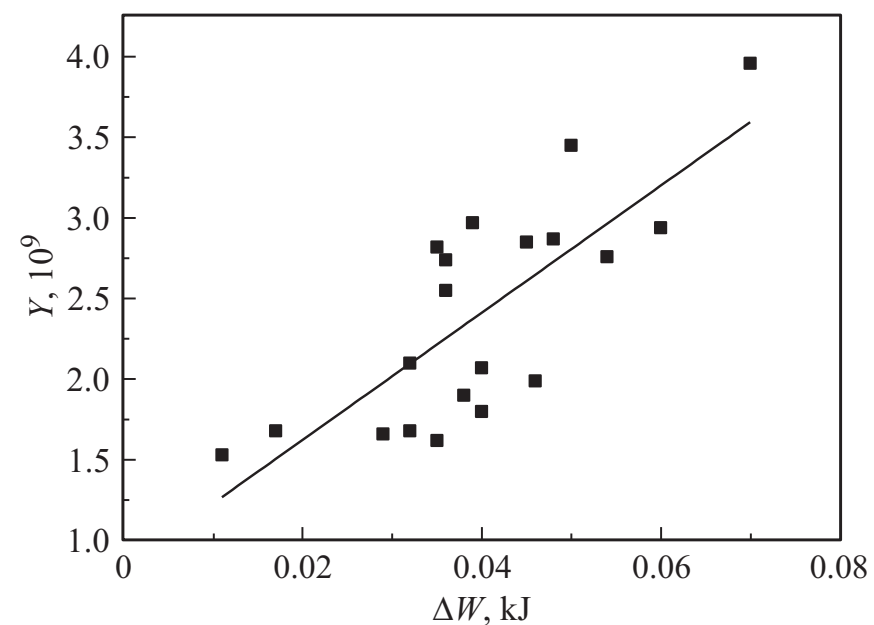

Рис. 5. Зависимость выхода нейтронов $Y$ от изменения энергии $\Delta W$ магнитного поля, $P=1.8 \cdot 10^{3} \mathrm{~Pa}$, коэффициент корреляции 0.76 .

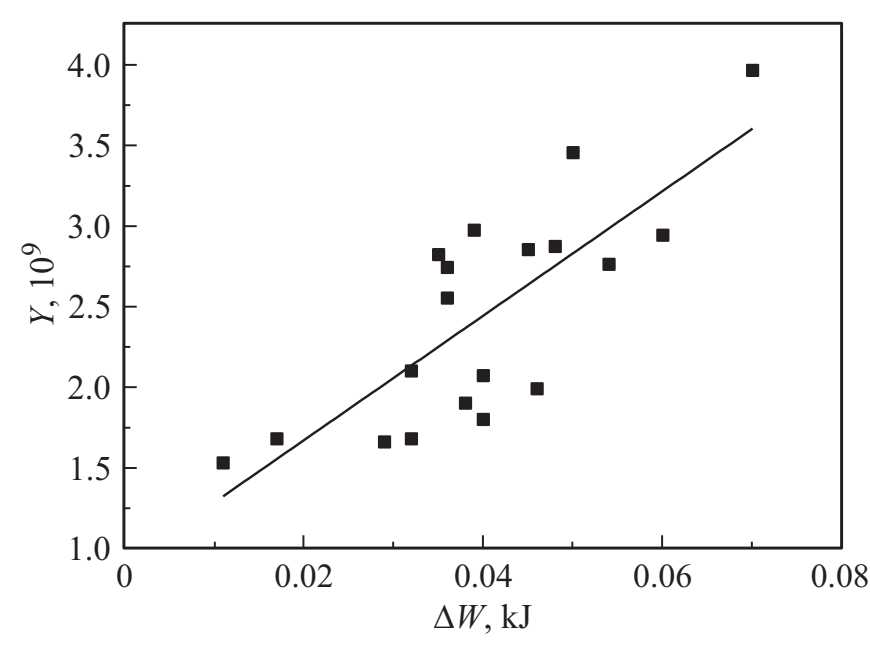

Рис. 6. Зависимость выхода нейтронов $Y$ от изменения энергии $\Delta W$ магнитного поля, $P=2.7 \cdot 10^{3} \mathrm{~Pa}$, коэффициент корреляции 0.76 .

Для исследованной плазмофокусной камеры обнаружена линейная связь выхода нейтронов с величиной изменения энергии $\Delta W$ магнитного поля разрядного контура в момент генерации нейтронов, а также влияние на указанную связь величины начального давления дейтерия в разрядной камере.

\section{Список литературы}

[1] Behbahani R.A., Aghamir F.M. // J. Appl. Phys. 2012. Vol. 111, P. 043304.

[2] Veloso V., Pavez C., MorenJ., Galaz V., Zambra M., Soto L. // J. Fusion Energy. 2012. Vol. 31. P. 30-37.

[3] Аблесимов В.Е., Долин Ю.Н., Пашко О.В., Цибиков З.С. // Физика плазмы. 2010. Т. 36. № 5. С. 436-439. 
[4] Аблесимов В.Е., Карпов Г.В., Цибиков З.С. // Физика плазмы. 2012. Т. 38. № 10. С. 890-893.

[5] Дудин В.И., Забиралов А.А., Курин С.А., Морозов И.В. // Проблемы физики высоких плотностей энергии. ХII Харитоновские науч. чтения. Саров. Доклады. 2010. С. 505-510.

[6] Mather J.W., Bottoms P.J. // Phys. Fluids. 1968. Vol. 11. N 3. P. 611-618. 